\title{
Numerical Models of Human Circulatory System under Altered Gravity: Brain Circulation
}

\author{
Chang Sung Kim, ${ }^{*}$ Cetin Kiris, $\dagger$ and Dochan Kwak† \\ NASA Ames Research Center, Moffett Field, CA 94035-1000 \\ Tim David + \\ University of Canterbury, Christchurch, New Zealand
}

\begin{abstract}
A computational fluid dynamics (CFD) approach is presented to model the blood flow through the human circulatory system under altered gravity conditions. Models required for CFD simulation relevant to major hemodymamic issues are introduced such as non-Newtonian flow models governed by red blood cells, a model for arterial wall motion due to fluid-wall interactions, a vascular bed model for outflow boundary conditions, and a model for auto-regulation mechanism. The three-dimensional unsteady incompressible Navier-Stokes equations coupled with these models are solved iteratively using the pseudocompressibility method and dual time stepping. Moving wall boundary conditions from the first-order fluid-wall interaction model are used to study the influence of arterial wall distensibility on flow patterns and wall shear stresses during the heart pulse. A vascular bed modeling utilizing the analogy with electric circuits is coupled with an auto-regulation algorithm for multiple outflow boundaries. For the treatment of complex geometry, a chimera overset grid technique is adopted to obtain connectivity between arterial branches. For code validation, computed results are compared with experimental data for steady and unsteady non-Newtonian flows. Good agreement is obtained for both cases. In six-type Gravity Benchmark Problems, gravity source terms are added to the Navier-Stokes equations to study the effect of gravitational variation on the human circulatory system. This computational approach is then applied to localized blood flows through a realistic carotid bifurcation and two Circle of Willis models, one using an idealized geometry and the other model using an anatomical data set. A threedimensional anatomical Circle of Willis configuration is reconstructed from human-specific magnetic resonance images using an image segmentation method. The blood flow through these Circle of Willis models is simulated to provide means for studying gravitational effects on the brain circulation under auto-regulation.
\end{abstract}

\section{Introduction}

During long-duration space missions, astronauts have to adapt themselves to the altered circumstance of microgravity. Blood circulation as well as body fluids distribution undergoes significant adaptation during and after space flight. Much study on physiological changes under weightlessness has been performed since the early days of the space program [1]. In particular, cardiovascular research in conjunction with the Space Shuttle program has included diverse physiological functions affected by the nervous system such as heart rate, blood pressure, hormone release, and respiration. The altered cardiac output due to decondi-

- RIACS Research Associate, AIAA Member

+ NASA Advanced Supercomputing (NAS) Applications Branch

"Professor, Department of Mechanical Engineering.

Copyright 92004 by the authors. Published by the American Institute of

Aeronautics and Astronautics, Inc. with permission. tioning during flight and readaptation after the flight will impact blood circulation in the human body. Especially, this altered blood supply in the brain and consequent oxygen supply to certain parts of the brain will make non-negligible impact on long-duration space flight. In flights on Earth, a gravitational force over $8 \mathrm{G}$ causes unconsciousness, socalled, blackout and a gravitational force below $-3 \mathrm{G}$ makes the retina engorged with blood, red-out. To assess the impact of changing gravitational forces on space and earth flights, it will be essential to quantify the flow characteristics in the brain under varying gravity conditions. Analysis of blood circulation in the brain as well as other parts of human body such as heart or kidney requires the capability to simulate blood flows in large arteries and capillaries.

In addition to the altered gravitational forces, specific shapes and connections of arteries in the brain, so-called Circle of Willis, vary in the human population $[2,3]$. Con- 
sidering the geometric variations, pulsatile unsteadiness, and moving walls, computational approach in analyzing altered blood circulation will thus offer an economical altemative to experiments in vivo or in vitro.

Technical challenges of the present study relevant to major hemodynamic issues can be summarized as i) anatomically complex geometry of vascular networks, ii) nonNewtonian modeling of the human blood, iii) distensible wall motion due to heart pulse, iv) vascular bed and autoregulation modeling for outflow boundary conditions, and v) gravitational effects. Recently, three-dimensional reconstruction techniques have been used to oblain the accurate anatomical vasculature from magnetic resonance imaging (MRI), magnetic resonance angiogram (MRA), and computed tomography (CT) [4-6]. Computational simulations coupled with these medical diagnostic imaging techniques can provide physicians with patient-specific information to predict the outcome of surgical procedures.

Blood flow characteristics are dominated by the presence of red blood cells since red blood cells constitute about 40 to 45 percent of human blood by volume. Red blood cell aggregation at low shear rates makes the apparent blood viscosity increase. Conversely, the viscosity decreases at higher shear rates due to red blood cell deformation. Three different experiments [7-9] show this so-called shearthinning phenomenon (Fig. 1). Various non-Newtonian models have been developed by curve-fitting to the experimental data [10]. It is commonly assumed that the apparent blood viscosity is a function of shear rate alone. In the present study, two non-Newtonian models are adopted among them and assessed through steady and unsteady nonNewtonian flow simulations.

Relative diameter change due to the heart pulse was found to be up to about 10 percent in common carotid arteries of young people [11]. It was also reported that the mean diameter change in large cerebral arteries (e.g., the carotid, the middle cerebral or the vertebral arteries) was less than 4 percent whereas the smaller arteries such as anterior cerebral arteries showed 21 percent diameter changes under moderate changes in the mean blood pressure $(30 \mathrm{mmHg}$ with $16 \mathrm{mmHg}$ deviation) [12]. On Earth, gravity pulls the blood to the feet. In standing posture, the blood pressure in the feet can be about $100 \mathrm{mmHg}$ higher than at the heart (approximately $100 \mathrm{mmHg}$ ) and 20 to $40 \mathrm{mmHg}$ lower in the brain. In space, blood pressure equalizes and becomes uniform throughout the human body. Consequently, the arterial wall distensibility due to gravity results in the fluid redistribution throughout the entire body. In the present study, a first-order approximation $[13,14]$ to the complex behavior of the arterial wall is extended to threedimensional problems by assuming that blood vessels have circular, thin and elastic walls. It has been found that if the wall is sufficiently thin, the computed results from finite element methods are very similar to that calculated by this approximation $[15,16]$.

To accomplish the simulation at a computationally manageable level, minor arteries such as arterioles, venules, and capillaries need to be truncated. At the truncated positions where flow information is hard to measure accurately in vivo, appropriate outflow boundary conditions are necessary. Alternative approaches using the analogy of arterial networks to electric circuits have been applied to supply the adequate boundary conditions for three-dimensional computations $[6,17]$. To simulate the feedback mechanism in peripheral resistance of the brain arterial tree, a numerical auto-reguiation model [18] was developed based on conirol theory and compared it with the experiment [19]. In the present study, a hybrid approach is presented for the problems with multiple outflow boundaries by combining the analogy with electric circuits and the mechanism of arteriolar auto-regulation.

There have been a few studies about computational simulations of blood circulation in the brain. Blood flow in patient-specific cases taken from MRA images was simulated as a planning tool for neuro-surgical and interventional procedures [4]. It was reported that the variants of arterial geometry and the proper functioning of the auto-regulation are crucial in determining the correct amount of blood supply to the brain $[18,20]$. To the authors' knowledge, the present study is one of the first simulations of the blood circulation in the human brain under altered gravity conditions using non-Newtonian flow models within deformable arterial walls.

\section{Computational Approach}

\section{Governing equations}

Blood flow through the heart and blood vesscls is unsteady, viscous, and incompressible. Fluids in the heart and larger vessels have been assumed to be Newtonian. For instance, the Newtonian flows through artificial heart devices have been simulated using the NS3D code $[21,22]$. In the present study, the flows of interest are assumed nonNewtonian to account for the shear thinning behavior of the human blood. The governing equations are the threedimensional, unsteady, incompressible Navier-Stokes equations coupled with non-Newtonian models. The solution algorithm is based on the method of artificial compressibility and dual time stepping. The resulting incompressible Navier-Stokes equations can be written in tensor notation form as:

$$
\begin{gathered}
\frac{\partial u_{k}}{\partial x_{k}}=0 \\
\frac{\partial u_{i}}{\partial t}+\frac{\partial}{\partial x_{j}}\left(u_{j} u_{i}\right)=-\frac{\partial p}{\partial x_{i}}+\frac{\partial \tau_{i j}}{\partial x_{j}}+g_{i}
\end{gathered}
$$

The shear stress tensor; $\tau_{i j}$ is defined as: 


$$
\tau_{i j}=\left\{\begin{aligned}
-2 \mu S_{i j}: \text { Newtonianflows } \\
-2 \eta(j) S_{i j}: \text { Non-Newtonianflows }
\end{aligned}\right.
$$

where $\mu$ and $\eta$ are molecular and apparent viscosity, respectively. $S_{i j}$ is the mean strain-rate tensor and $\dot{\gamma}$ is the shear rate defined as a function of the second invariant of $S_{i j}$ in three-dimensional problems:

$$
\begin{gathered}
S_{i j}=\frac{1}{2}\left(\frac{\partial i_{i}}{\partial x_{j}}+\frac{\partial z s_{j}}{\partial x_{i}}\right) \\
\dot{\gamma}=2 \sqrt{\Pi_{S}}=\sqrt{2\left(S_{i j} S_{i j}-S_{k k}^{2}\right)}
\end{gathered}
$$

The gravity force terms, $g_{i}$ are added to Eq.(2) to take gravitational effects into account. With the reference pressure at heart level, the blood pressure gradient due to height difference is also added to boundary conditions.

\section{Non-Newtonian models}

Two different types of non-Newtonian models are implemented into the INS3D code [23] and evaluated through steady and unsteady computations. One is a Carreu-Yasuda model and the other is an extended Casson model. A Carreau-Yasuda model in Eq. (6) describes the shear thinning behavior of blood flows with asymptotic apparent viscosities at zero and infinite shear rates:

$$
\frac{\eta-\eta_{\infty}}{\eta_{0}-\eta_{\infty}}=\left[1+(\lambda \dot{\gamma})^{a}\right]^{n-1} a
$$

where $\lambda$ and $a$ define the width of the transition region from Newtonian to power-law region, and $n-1$ is power-law slope as shown in Fig. 1. The constitutive parameters for the human blood are given by:

$$
\begin{aligned}
& \eta_{\infty}=0.00348 \mathrm{~Pa} \cdot s, \eta_{0}=0.1518 \mathrm{~Pa} \cdot s \\
& \lambda=40.0 s, a=2.0, n=0.356
\end{aligned}
$$

For an extended Casson model,

$$
\eta=\left(\frac{1}{\dot{\gamma}}\left[C_{1}(H t)+C_{2}(H t) \sqrt{\dot{\gamma}}\right)^{p}, \eta_{\max }\right)
$$

where $H t$ is the hematocrit, $C_{1}$ and $C_{2}$ are coefficients determined for $H t=40$ percent as $C_{1}=0.2\left(d y n / \mathrm{cm}^{2}\right)^{1 / 2}$ and $C_{2}=0.18\left(\mathrm{dyn} \cdot \mathrm{s} / \mathrm{cm}^{2}\right)^{1 / 2}$ based on the experimental data [8]. To avoid extreme values at lower shear rates, the apparent viscosity is confined within $\eta_{\max }=0.1518 \mathrm{~Pa} \cdot \mathrm{s}$.

\section{Deformable wall model}

For moving wall boundaries, a first-order fluid-wall interaction model for the arterial wall distensibility is adopted based on the assumption of a thin-walled and linearly elastic channel. Compared with finite element methods $[15,16]$, this approach was found to be a standard first-order approximation to the complex behavior of the arterial wall [14]. Embedding in and tethering to surrounding structures is thought to reduce the arterial longitudinal motion to a minimum [24]. In this wall model, blood vessels are assumed to have circular, thin and elastic wails with negiigible longitudinal motion. The increments of blood vessel radius, $r$ are given by:

$$
\frac{\Delta r_{i}}{r}=\frac{1-v^{2}}{E} \frac{r}{h}\left(p_{w}-\bar{p}_{w}\right)_{i}=D_{w}\left(p_{w}-\bar{p}_{w}\right)_{i}
$$

where $p_{w}$ and $\bar{p}_{w}$ are transmural and reference pressure at wall, and the arterial wall distensibility $D_{w}$ is defined as:

$$
D_{w}=\frac{1-v_{P}^{2}}{E} \frac{r}{h}
$$

where $h$ is the wall thickness, $E$ and $v_{p}$ are the elastic modulus and Poisson's ratio of the arterial wall, respectively. For deformable walls, non-slip boundary conditions are replaced by moving wall boundary conditions using the Eq. (9) at each physical time step.

\section{Vascular bed modeling}

To make the problem computationally manageable, minor arteries such as arterioles, venules and capillaries need to be truncated. Equivalent outflow boundary conditions should be imposed at the truncated positions. There is an analogy between arterial networks and electric circuits as shown in Fig. 2. A truncated artery is assumed to divide into $N$ branches of the same size, for instance, $N$ equals two for bifurcation and three for trifurcation. Under this assumption, the outflow boundary conditions, especially for pressure, can be approximately determined by utilizing the electric circuit analogy and Poiseuille's theorem $[25,4]$ :

$$
\dot{Q}=\frac{\pi r^{4}}{8 \mu L} \Delta p \Rightarrow \dot{Q}=\frac{\Delta p}{\mathrm{R}}, \dot{Q}_{k+1}=\frac{1}{N} \dot{Q}_{k}
$$

where $\dot{Q}$ is mass flow rate, $p$ is pressure, and $\mathrm{R}$ is flow resistance. At the $k^{\text {h }}$ bifurcation (or trifurcation), its mass flow rate is $N$ times of flow rate through the $(k-1)^{\text {st }}$ branches. Assuming that the flow resistance ratio $f$ is constant, the pressure drop at each level can be expressed in a geometri- 
cal series form:

$$
\begin{gathered}
\Delta p_{k+1}=\mathrm{R}_{k+1} \dot{Q}_{k+1}, \Delta p_{k}=\mathrm{R}_{k} \dot{Q}_{k} \\
\Delta p_{k+1}=\frac{1}{N}\left(\frac{\mathrm{R}_{k+1}}{\mathrm{R}_{k}}\right) \Delta p_{k}=f \Delta p_{k}, f=\frac{1}{N}\left(\frac{\mathrm{R}_{k+1}}{\mathrm{R}_{k}}\right) \\
\Delta p_{\text {Total }}=\sum_{k=1}^{\infty} \Delta p_{k}=\frac{1}{1-f} \Delta p_{1}=\frac{8 \pi \mu L_{1}}{(1-f) A_{1}{ }^{2}} \dot{Q}_{1} \\
\therefore \Delta p=\frac{8 \pi \mu L}{(1-f) A^{2}} \dot{Q}
\end{gathered}
$$

where $A$ and $L$ are sectional area and length of the vessel, respectively.

\section{Arteriolar auto-regulation}

The flow resistance ratio $f$ in Eq.(12) is usually unknowin and thus should be given to avoid an unrealistic pressure difference between a truncated position and the vascular bed. The arteriolar bed varies its flow resistance dynamically by dilating or constricting in order to maintain the constant blood flow within a certain range of the perfusion pressure. To model this auto-regulation mechanism in the arteriolar bed, as well as to determine the adequate value of $f$, the arteriolar auto-regulation (AAR) algorithm is developed here by coupling the vascular bed modeling with the auto-regulation mechanism:

$$
\begin{gathered}
p_{e}^{n+1}=p_{r e f}+\frac{8 \pi \mu L}{\left(1-f^{n+1}\right) A^{2}} \dot{Q}_{e}^{n+1}=p_{r e f}+R_{e}^{n+1} \dot{Q}_{e}^{n+1} \\
R_{e}^{n+1}=\frac{8 \pi u L}{\left(1-f^{n+1}\right) A^{2}}, f^{n+1}=1-0.5 / a^{n+1} \\
a^{n+1}=a^{n}+k_{t} \frac{\left(\dot{Q}_{e}^{n+1}-\dot{Q}_{r e f}\right)}{\dot{Q}_{r e f}} \\
k_{t}=\alpha \Delta t / T
\end{gathered}
$$

where $\alpha$ is given by $3.6 \sim 8.0$ for the adequate autoregulatory response, and $T$ is a cyclic period of the heart beat.

\section{MPI-OpenMP parallel processing}

An MPI-OpenMP hybrid version of the INS3D code [23] has been extended with the above numerical approaches and validated through comparison with experimental data for both steady and unsteady non-Newtonian cases. Computational experiments have been conducted utilizing the NASA Advanced Supercomputing (NAS) facilities at NASA Ames Research Center.

\section{Code validation}

\section{Results and Discussion}

The steady non-Newtonian flow in a carotid bifurcation illustrated in Fig. 3 was calculated as the first validation problem. Anatomically, a pair of common carotid arteries (CCA) arise from the ascending aorta of the heart and connect to the brain through the neck. CCA are divided into internal (ICA) and external carotid arteries (ECA), respectively. Computations are conducted with the same flow condition of the experiment [26]. In the experiment, a blood analogy fluid (KSCN-X; KSCN-Xanthan gum solution) was used to mimic the shear thinning property of blood. The constitutive parameters of the Carreau-Yasuda model in Eq. (7) are modified based on the experimental data as following:

$$
\begin{aligned}
& \eta_{\infty}=0.0022 \mathrm{~Pa} \cdot s, \eta_{0}=0.022 \mathrm{~Pa} \cdot s, \\
& \lambda=0.11 s, a=0.644, n=0.392
\end{aligned}
$$

Likewise, a parameter of the extended Casson model is modified as $C_{1}=0.4\left(d y n / \mathrm{cm}^{2}\right)^{1 / 2}$ for this analogy fluid.

The Reynolds number based on the CCA diameter is 270 , and the flow division ratio of ECA over CCA is 0.45 . The inflow in the CCA is assumed fully developed and multiple outflow boundary conditions for ICA and ECA are determined using the AAR algorithm in Eq. (13) based on the flow division ratio. Figure 4 (a) and (b) show the comparison of axial velocity profiles in the ICA using two different non-Newtonian models. The results of the INS3D with chimera overset grids are compared with those of IFANS3D [27] with multi-block grids, and also compared to the experimental data. Axial velocity profiles are skewed towards the flow divider and the adverse pressure gradient with the increase of the sectional area results in a secondary flow pattern in the sinus of the ICA. The flow reaccelerates after passing through the maximum diameter region. Since two non-Newtonian models gave a similar result, the CarreauYasuda model was mainly used hereafter in all the computations of non-Newtonian flows.

For unsteady code validation, the pulsatile nonNewtonian flow in a $90 \mathrm{deg}$ circular tube was simulated with the same flow condition of the experiment [28]. The same blood analogy fluid used in the previous steady case was also used in this case. The Reynolds number based on the tube diameter and diastole velocity is 300 and Womersley number is 7 . The tube radius and its centerline radius of curvature are 4 and 24 millimeters, respectively. For pulsatile inflow boundary conditions, the experimental waveform was successfully regenerated using twelve harmonics based on the Fourier theorem as shown in Fig. 5. Computed results are compared with the experimental data at three different phases: end diastole, peak systole, and begin diastole. Figure 6 shows the comparison of axial velocity profiles on the plane of symmetry and the plane perpendicular to the symmetry plane. The computed results show a good agreement with the experimental data at all three phases. 


\section{Gravity Benchmark Problems}

Six-type Gravity Benchmark Problems (GBP) were originally presented to provide the fundamental understanding of gravitational effects on the human circulatory system. As shown in Fig. 7, GBP-0 stands for microgravity and GBP-1 represents the human body in supine posture under normal gravity on Earth. Among six problems, let us discuss GBP-2 and 3 cases representing the upper and lower part of the human body in standing posture, respectively. Test model is a U-tube with a diameter of 8 millimeters and a curvature ratio of 4 . The fluid is assumed Newtonian with a viscosity of $3.5 \mathrm{cPoise}$ and the Reynolds number based on the tube diameter is 169 . Figure 8 shows a comparison of axial velocity profiles between rigid- and deformable-wall cases at the 90 deg station. A first-order fluid-wall interaction model in Eq. (9) was adopted for wall moving boundary conditions, and the wall distensibility factor is given about five times larger than that of normal arteries to bring gravitational effects into relief. Even though not all shown here, the gravitational effect on the intemal flow within rigid wall was found to be minor.

Table 1. Comparison of required power for GBP-2 and 3.

\begin{tabular}{|c|c|c|c|c|}
\hline & Wall & $\begin{array}{c}\Delta p \\
(P a)\end{array}$ & $\begin{array}{c}Q \\
\left(\mathrm{~cm}^{3} / s\right)\end{array}$ & $\begin{array}{c}\Delta p Q \\
(\mu \mathrm{W})\end{array}$ \\
\hline \multirow{2}{*}{ GBP-2 } & Rigid & 21.4151 & 3.5186 & 75.3512 \\
\cline { 2 - 5 } & Deformable & 24.8279 & 3.5186 & 87.3595 \\
\hline \multirow{2}{*}{ GBP-3 } & Rigid & 21.0905 & 3.5186 & 74.2090 \\
\cline { 2 - 5 } & Deformable & 19.1109 & 3.5186 & 67.2438 \\
\hline
\end{tabular}

For GBP-2W (GBP-2 with deformable wall), however, tube diameter is contracted by about $5 \% \mathrm{D}$ because of the decreased pressure due to gravity. Conversely for GBP-3W, the diameter is expanded by $5 \% \mathrm{D}$ because of the increased pressure. As flow resistance is, according to Poiseuille's formula, inversely proportional to the fourth power of tube radius, GBP-2W requires about 16 percent more power and GBP-3W requires about 10 percent less power as shown in Table 1, where $\Delta p$ denotes pressure difference between inflow and outflow.

GBP provide a preliminary knowledge that gravity has considerable effects on deformable wall motion and consequent flow patterns in the human circulatory system.

\section{Effect of arterial wall distensibility}

Pulsatile blood flow through the same carotid bifurcation model used for code validation was computed with the mean flow rate of $8 \mathrm{ml} / \mathrm{s}$ in the common carotid artery (CCA) with a Reynolds number of 388. Figure 9 shows a moving wall grid at maximum displacement together with the baseline rigid-wall grid. For this carotid model, the arterial wall thickness is 0.3 millimeter for the CCA, 0.24 and 0.21 millimeter for the internal (ICA) and external carotid artery (ECA), respectively. A smooth connectivity in wall thickness between the CCA and its branches is enforced. The elastic modulus $E$ is given by $3.0 \times 10^{6}$ dynes $/ \mathrm{cm}^{2}$ and the Poisson's ratio $v_{p}$ is 0.49 , which represents a nearly incompressible isotropic wall property $[14,15]$. The bifurcating apex is geometrically constrained to prevent unrealistic rigid body motion. Figure 10 shows particle iraces at line differentit positions in time: (a) systulic acederation, (b) systolic deceleration, and (c) minimum flow rate. During a pulse cycle, the distensible wall case shows a maximal displacement of about 8 percent of vessel diameter around the bifurcating apex. At the systolic deceleration phase, the increased vessel diameter reduces the axial velocity profiles compared with the rigid wall results. Much like the steady-state results, a strong skewing toward the flow divider walls occurs. After the peak systole, a secondary reversed flow occurs and extends along the sinus until the diastole begins. The wall distensibility due to the pulse alleviates the amplitude of wall shear stress locally up to 16 percent compared with the results under the rigid wall assumption as shown in Fig. 11. The temporal wall shear stress at the point $\mathrm{C}$ in the ICA sinus indicates the progress of massive flow separation and reattachment on the outer wall of the sinus during $t / T=0.22 \sim 0.54$ in both distensible and rigid wall cases.

In addition, the effect of wall distensibility under different gravity conditions is presented in Fig. 12. Computed results for microgravity (or approximately supine posture under normal gravity), standing and hand-standing conditions are compared. For the standing case, the normal one$G$ gravity force (or $1 G$ ) is applied downward. For the handstanding case, the gravity force is applied toward the head, i.e., it is negative (or $-1 G$ ). The relative diameter change $\left(D_{-1 G}-D_{1 G}\right) / D_{1 G}$ is up to 14 percent during the systolic deceleration phase, which corresponds to a 41 percent reduced vessel resistance from Poiseuille's theorem in Eq. (11). Wall shear stress distribution in Fig. 13 indicates that the reversed flow zone in the ICA sinus becomes narrower as the diameter increases. It is observed that the altered gravity has a significant influence on fluid-wall interactions throughout the pulse cycle.

\section{Blood circulation in the human brain}

There are two arterial pairs that supply blood to the brain. One pair is the intermal carotid arteries (ICA) and the other is the vertebral arteries. The vertebral arteries are distally combined into the basilar artery (BA) that ends by dividing into the two posterior cerebral arteries (PCA). Two ICAs and $\mathrm{BA}$ are connected to an important part of the brain, the so-called Circle of Wills (CoW). CoW sits on the base of 
the brain. Its main function is to distribute blood evenly throughout the brain.

\section{Collateral circulation under auto-regulation}

To provide a fundamental understanding of the mechanism of collateral circulation under auto-regulation, an idealized CoW configuration was designed based on anatomical measurements $[2,3]$ with minor arteries truncated. Figure 14 shows a chimera overset grid with ten domains for this idealized configuration, which results in a total of 0.3 million grid points. The mean flow rate in the ICA is 3.5 $\mathrm{ml} / \mathrm{s}$ and the Reynolds number is 240 based on the ICA diameter.

When one of the main arteries in the brain is stenosed or even missing, the distal smaller arteries can receive blood from the other arteries through $\mathrm{CoW}$. To simulate this interesting mechanism of "collateral circulation" under autoregulation, the left IC.A is presumed 20 percent stenosed. This means that only 80 percent of the normal supply of blood is delivered to CoW through the left ICA as indicated in Fig. 15. Unlike the balanced configuration case, the mass flux through the posterior communicating arteries (PCoA) is considerably increased to compensate for the deficiency in the left middle cerebral artery (MCA). On the other hand, the mass flux through the proximal part ( $A 1$ segment) of the left anterior cerebral artery (ACA) is decreased by 26 percent in order to distribute the blood as evenly as possible. Figure 16 shows the time-dependent auto-regulatory process using the AAR algorithm introduced in Eq. (10). It is observed that this AAR algorithm is robust and consistent for a wide range of physical time steps $(d t=0.05 \mathrm{~T}$ to $0.25 T$ ). About 10 seconds after the sudden stenosis in the left ICA, the left MCA and ACA have regained their reference flow rates. The present simulation shows a good example of collateral circulation in the brain under autoregulation.

\section{Effects of altered gravity}

An anatomically realistic CoW geometry was threedimensionally reconstructed from human-specific magnetic resonance (MR) angiography (MRA, provided by $T$. David) using image segmentation techniques as illustrated in Fig. 17. The raw $M R$ images were converted to the $R G B$ graphic file format for efficient numerical treatments. After extracting the segments of interest by filtering the voxels with intensities below a certain threshold, a segment outlining algorithm was used to display the extracted objects on each sectional layer with very little computer memory. Consequently, a chimera overset grid system with thirty-one domains was generated for this geometry as shown in Fig. 18. To demonstrate the effects of altered gravity, a computed result under a normal $1 G$ gravity (i.e., standing posture) was compared with results for $-1 G$ (i.e., hand-standing posture). For both cases, the total inflow rate through the left and right ICAs and the BA is assumed the same because the auto-regulation is known to supply constant blood flow regardless of altered pressure to a certain extent. For distensible wall motion, local changes of the wall distensibility factor, $D_{w}$ in Eq. (10) were given by introducing different ratios of vessel radius and wall thickness to each arterial component. Based on the experimental measurements [12], large cerebral arteries (internal carotid, vertebral artery) are assumed to have about five times smaller $D_{w}$ compared with smaller arteries such as anterior cerebral arteries (ACA). The wall distensibility at the arterial conjunctions was neglected to avoid the unrealistic rigid body motion. Smooth transition in wall distensibility factor between each conjunction and its arterial branch was given. Figures 19 and 20 show the blood flow distribution through the realistic CoW configuration under $1 G$ and $-1 G$, respectively. In both cases, the mass flux through PCOA is very low. For the $1 G$ case, the biood pressure is decreased by about 25 mmHg. This causes about 16 percent contraction in ACA diameter. On the other hand, the increased pressure due to the hand-standing posture causes a maximum increase in ACA diameter up to 15 percent. Overall the magnitude of flow velocity is decreased to maintain the same flow rate with IG case.

\section{Summary}

Numerical models based on computational fluid dynamics were developed to simulate the local hemodynamics in the human circulatory system, and then applied to a realistic carotid artery and brain arterial trees. The solution procedure for non-Newtonian flow computations was validated through the comparative study with steady and unsteady experiments. Good agreement was obtained for both cases. For the realistic carotid artery model, the wall distensibility had a quantitative influence on deformable wall motions and wall shear stress distribution under altered gravity. In addition, fundamental understandings of the collateral circulation under auto-regulation and the gravitational effect on fluid-wall interactions were obtained using both ideal and realistic Circle of Willis configurations. The AAR algorithm based on the vascular bed modeling and autoregulation was assessed and found to be most useful for the problems with multiple outflow boundaries. The present solution procedure based on parallel computing has shown its ability to model the multi-scale hemodynamics for various aspects of the human circulatory system.

\section{Acknowledgements}

The first author would like to thank Dr. David Kao at NASA Ames Research Center for the helpful discussion about post-processing images. Computer resource was provided by the NASA Advanced Supercomputing (NAS) Division at NASA Ames Research Center. 


\section{References}

1) http://spaceresearch.nasa.gov/research_projects/biomedi cal.html

2) Alpers, B. J., Berry, R. G, and Paddison, R M., "Anatomical Studies of the Circle of Willis in Normal Brain," Arch. Neurol. Psychiatry, 1959, Vol. 81, pp. 409-418.

3) Gray, H., "Anatomy of the Human Body," 20th Edition by Lewis, W.H., Philadelphia: Lea \& Febiger, 1918, New York: Bartleby.com, 2000.

4) Cebral, J. R., Lohner, R., and Burgess, J., "Computer Simulation of Cerebral Artery Clipping: Relevance to Aneurysm Neuro-Surgery Planning," Proc. ECCOMAS, Sep. 11-14, Barcelona-Spain, 2000.

5) Taylor, C. A., Draney, M. T., Ku, J. P., Parker, D., Steele, B. N., Wang, K., and Zarins, C. K., "Predictive Medicine: Computational Techniques in Therapeutic DecisionMaking," Computer Aided Surgery, Vol. 4, No. 5, 1999. pp. 231-247.

6) Quarteroni, A., Tuveri, M., and Veneziani, A., "Computational Vascular Fluid Dynamics: Problems, Models and Methods," Computing and Viszalization in Science, Vol. 2, No.4, 2000, pp. 163-197.

7) Chien, S., "Biophysical Behavior of Red Blood Cells in Suspensions," The Red Blood Cell, Vol. II, Edited by Surgenor, D. M., Academic Press, New York, 1975, pp. 1031-1133.

8) Merrill, E. W., "Rheology of Blood," Physiology Reviews, Vol. 49, 1969, pp. 863-888.

9) Thurston, G. B., "Rheological Parameters for the Viscosity, Viscoelasticity and Thixotropy of Blood, Biorheology, Vol. 16, 1979, pp. 149-162.

10) Zhang, J.-B. and Kuang, Z.-B., "Study on Blood Constitutive Parameters in Different Blood Constitutive Equations," Journal of Biomechanics, Vol. 33, 2000, pp. 355 360.

11) Reneman, R. S., Merode, T. van, Hick, K., Muijtjens, A. M. M., and Hoeks, A. P. G., "Age-related Changes in Carotid Artery Wall Properties in Man," Vltrasound in Medicine and Biology, Vol. 12, 1986, pp. 465-471.

12) Giller, C. A., Bowman, G., Dyer, H., Mootz, L., and Krippner, W., "Cerebral Arterial Diameters During Changes in Blood Pressure and Carbon Dioxide During Craniotomy," Neurosurgery, Vol. 32, No. 5, 1993, pp. 737-741.

13) Caro, C. G., Pedley, T.J., Schroter, R. C., and Seed, W. A., "The Mechanics of the Circulation," Oxford University Press, Oxford, 1978.

14) Steinman, D. A. and Ethier, C. R, "Effect of Wall Distensibility on Flow in a Two-Dimensional End-to-Side Anastomosis," ASME Journal of Biomechanical Engineering, Vol. 116, 1994, pp. 294-301.

15) Perktold, K and Rappitsch, G, "Computer Simulation of Local Flow and Vessel Mechanics in a Compliant Ca- rotid Artery Bifurcation Model," J. Biomechanics, Vol 28, No. 7, 1995, pp. 845-856.

16) Zhao, S. Z., Ariff, B., Long, Q., Hughes, A. D., Thom, S. A., Stanton, A. V., and Xu, X. Y., “Inter-individual Variations in Wall Shear Stress and Mechanical Stress Distributions at the Carotid Artery Bifurcation of Healthy Humans," Journal of Biomechanics, Vol. 35, 2002, pp. 1367-1377.

17) Formaggia, L., Gerbeau, J. F., Nobile., F., and Quarteroni, A., "Numerical Treatment of Defective Boundary Conditions for the Navier-Stokes Equations," SIAM $J$. Numer Anal. Vol. 40 , ivo. i, $200 \hat{2} 2$, pp. $376-40 \hat{1}$.

18) Ferrandez, A., David, T., and Brown, M. D., "Numerical models of Auto-regulation and Blood Flow in the Cerebral Circulation," Computer Methods in Biomechanics and Biomedical Engineering, Vol. 5 (1), 2002, pp. 7-20.

19) Newell, D. W., Aaslid, R, and Lam, A., Mayberg, T. S., and Winn, H. R., "Comparison of Flow and Velocity During Dynamic Autoregulation Testing in Humans," Stroke, Vol. 25, No. 4, 1994, pp.793-797.

20) Ferrandez, A. and David, T., "Computational models of blood flow in the Circle of Willis," Computer Methods in Biomechanics and Biomedical Engineering, Vol. 4, No. 1, 2000, pp. 1-26.

21) Rogers, S. E., Kwak, D., and Kiris, C., "Steady and Unsteady Solutions of the Incompressible Navier-Stokes Equations," AIAA Journal, Vol. 29, No. 4, 1991, pp. 603610.

22) Kiris, C., Kwak, D., Rogers, S., and Chang, I.-D., "Computational Approach for Probing the Flow Through Artificial Heart Devices," ASME Journal of Biomechanical Engineering, 1997, Vol. 119, pp. 452-460.

23) Kiris, C. and Kwak, D., "Aspects of Unsteady Incompressible Flow Simulations," Computers \& Fluids, Vol. 31, 2002, pp. 627-638.

24) Milnor, W. R., "Hemodynamics," 2nd ed., The Williams \& Wilkins Co., 1989.

25) Nichols, W. W. and O'Rourke, M. F., "McDonald's Blood Flow in Arteries: Theoretical, experimental and clinical principles," $4^{\text {th }}$ Edition, Amold, London, 1998.

26) Gijsen, F. J. H., van de Vosse, F. N., and Janssen, J. D., "The Influence of Non-Newtonian Properties of Blood on the Flow in Large Arteries: Steady Flow in a Carotid Bifurcation Model," Journal of Biomechanics, 1999, Vol. 32, pp. 601-608.

27) Kim, C. S., "Sensitivity Analysis for the Navier-Stokes Equations with Two-Equation Turbuience Models and Its Applications," Ph.D. Dissertation, Seoul National University, Seoul, Korea, 2001.

28) Gijsen, F. J. H., Allanic, E., van de Vosse, F. N., and Janssen, J. D., "The Influence of Non-Newtonian Properties of Blood on the Flow in Large Arteries: Unsteady Flow in a 90 Degree Curved Tube," Journal of Biomechanics, 1999, Vol. 32, pp. 705-713. 


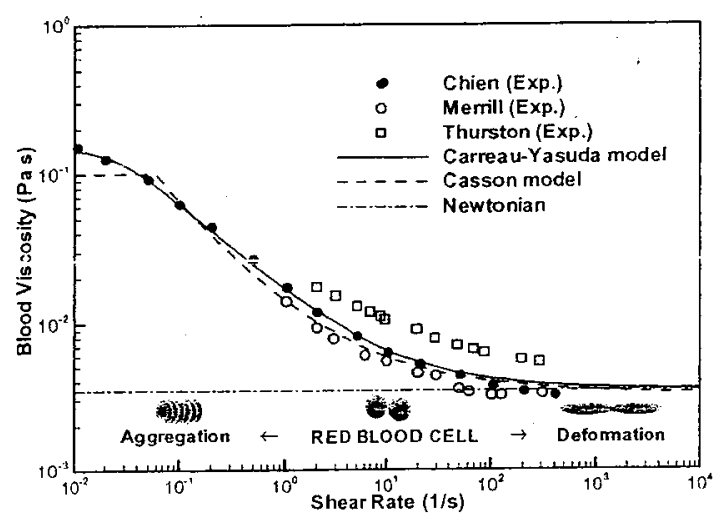

Fig. 1 Variation of blood viscosity due to shear rate.

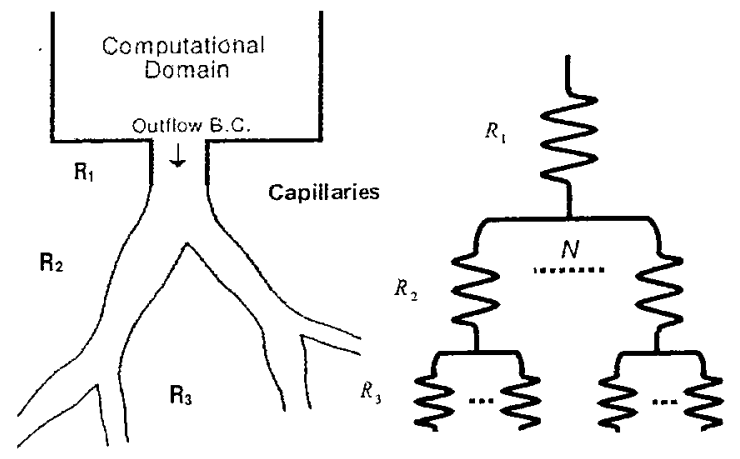

Fig. 2 Analogy of arterial network to electric circuit.

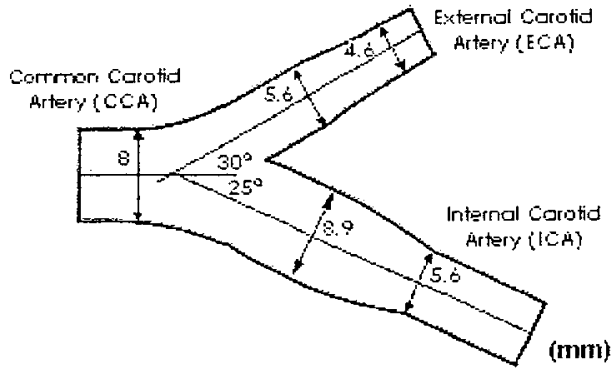

Fig. 3 Schematic definition of a carotid arterial bifurcation.

Fig. 2 Analogy of arterial network to electric circuit.

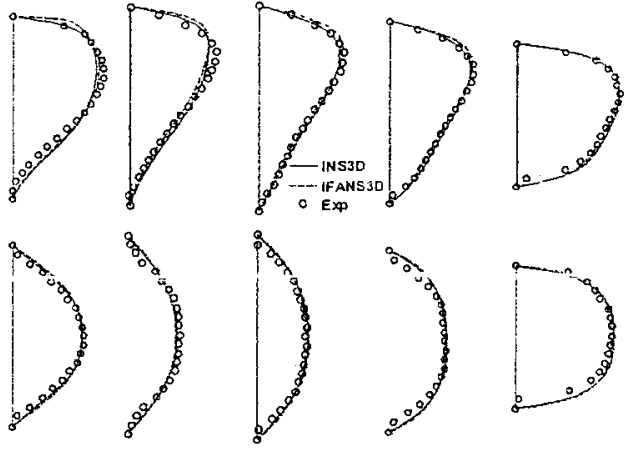

(a) Carreau-Yasuda model
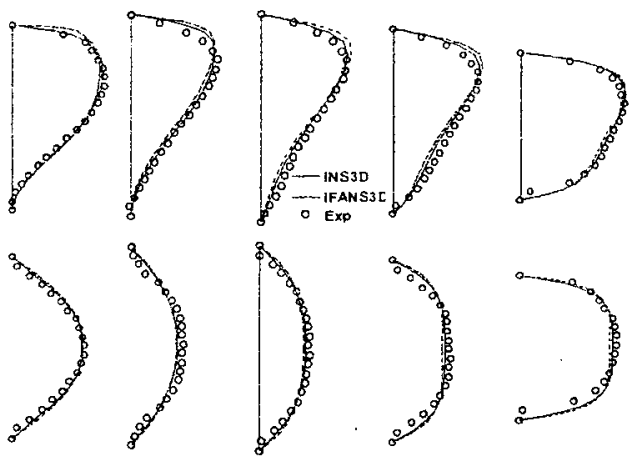

(b) Casson type model

Fig. 4 Axial velocity profiles on the symmetry (upper) and its perpendicular plane (lower) in the ICA.

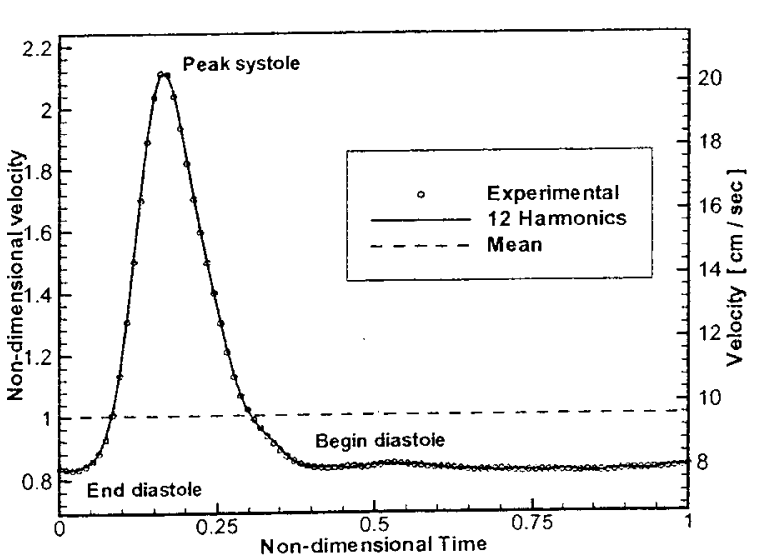

Fig. 5 Inflow waveform regenerated using 12 harmonics. 

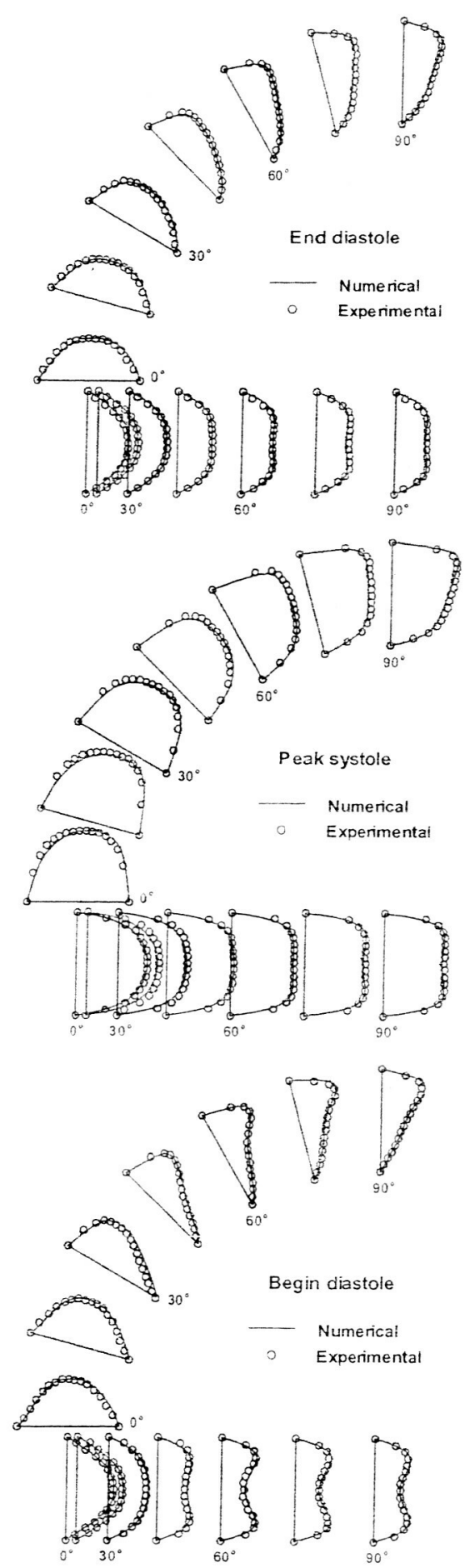

Fig. 6 Comparison of a xial velocity profiles between computation and experiment at three different phases.

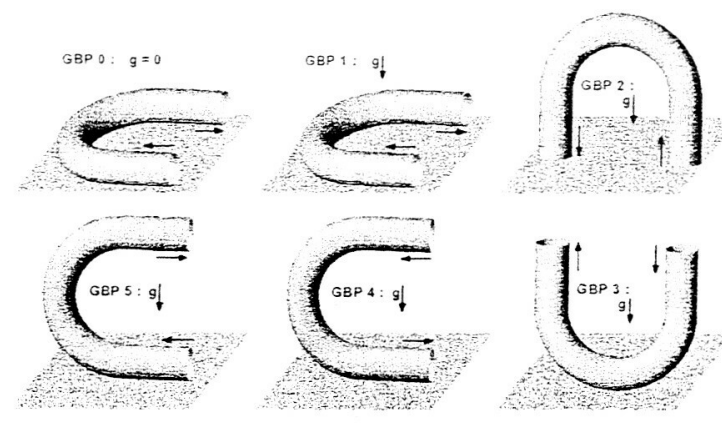

Fig. 7 Six-type gravity benchmark problems (GBP).
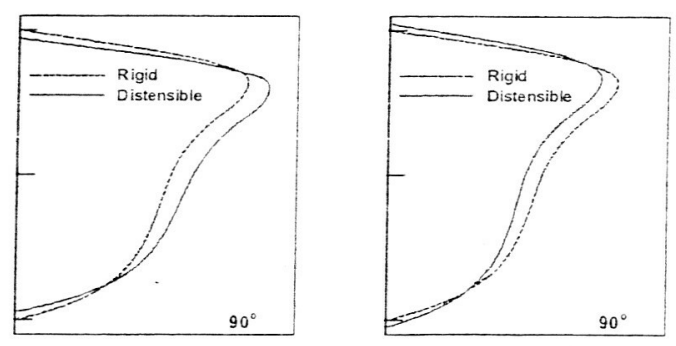

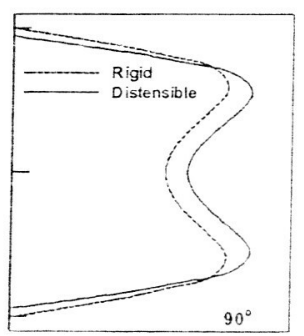

(a) GBP-2

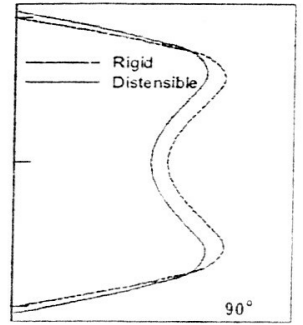

(b) GBP-3
Fig. 8 Comparison of axial velocity profiles at $90 \mathrm{deg}$ station between rigid-and distensible-wall cases.

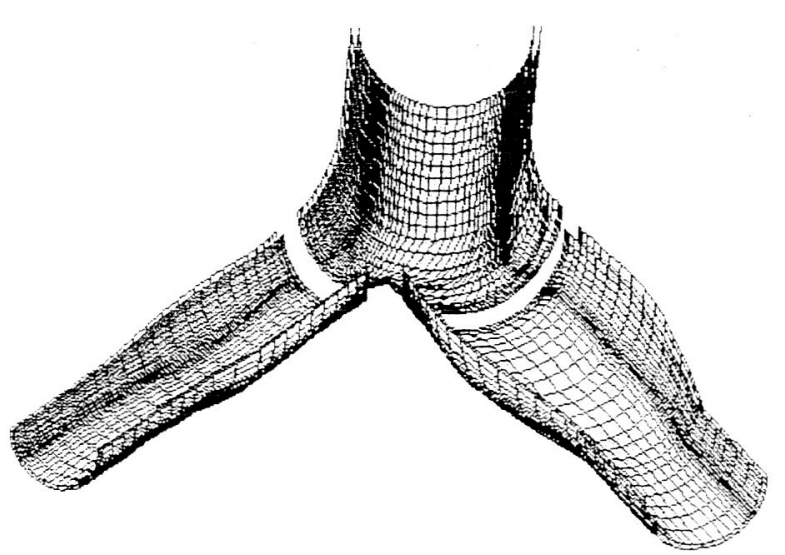

Fig. 9 Distensible wall motion due to fluid-wall interaction. (inner - rigid wall, outer - distensible wall) 


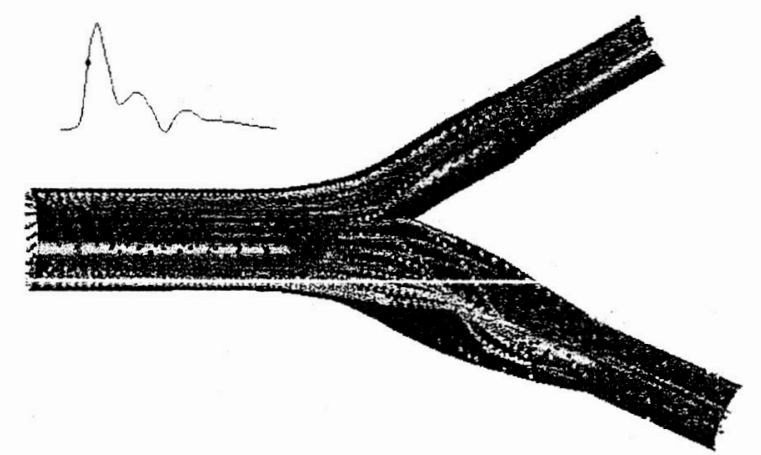

(a) Systoiic acceleration phase $(t / T=0.28)$

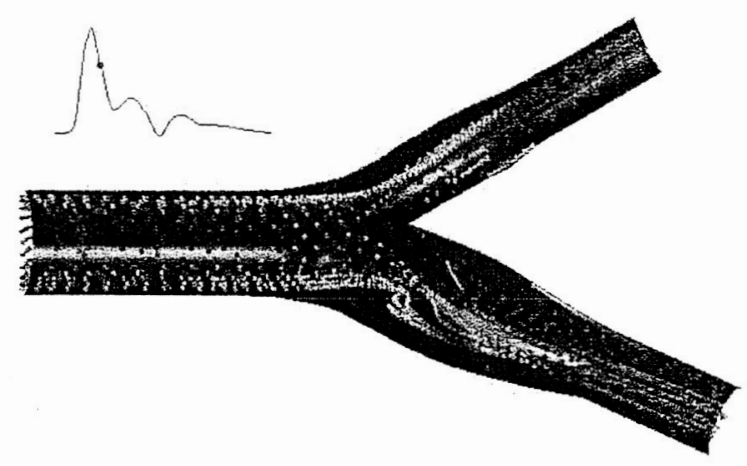

(b) Systolic deceleration phase $(t / T=0.36)$

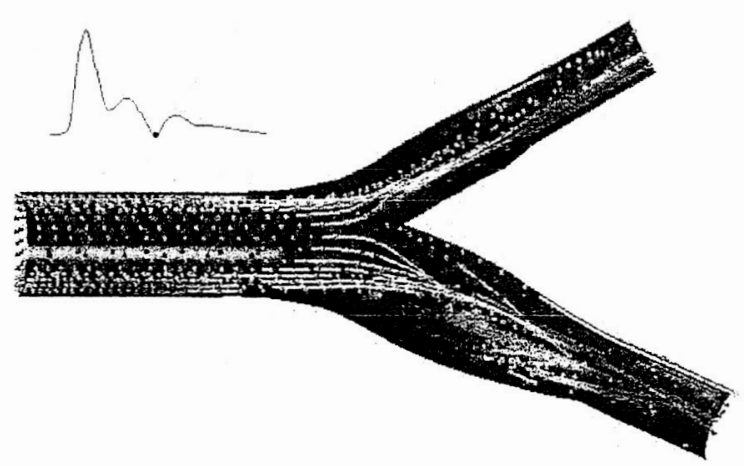

(c) Minimum flow rate phase $(t / T=0.58)$

Fig. 10 Streaklines through a carotid arterial bifurcation at three different phases in pulsatile flow rate.

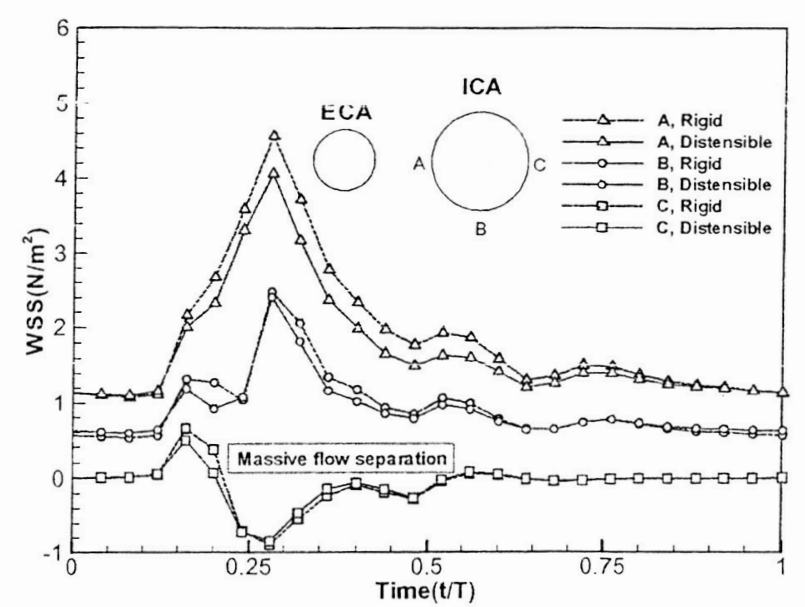

Fig. 11 Temporal wall shear stress during the pulse cycle at three different points around the ICA sinus.

(ICA: Internal carotid artery, ECA: External carotid artery)

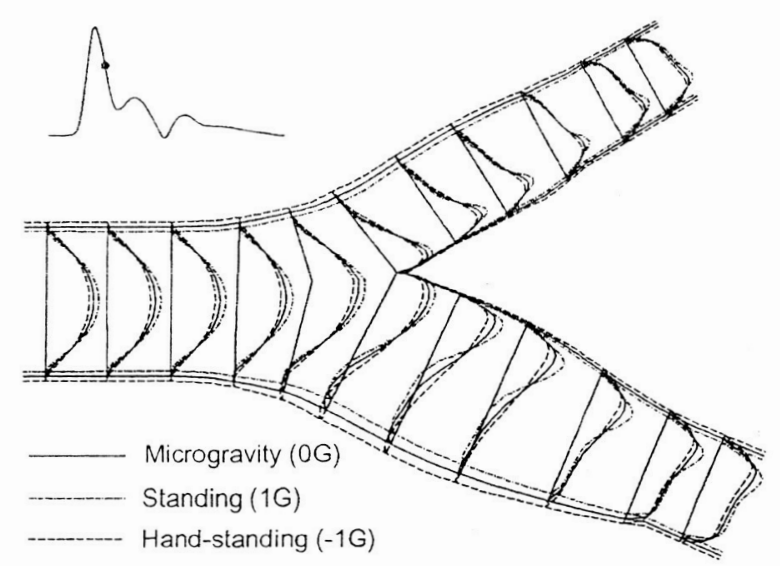

Fig. 12 Gravitational effects on wall motion and axial velocity profiles at systolic deceleration phase. 


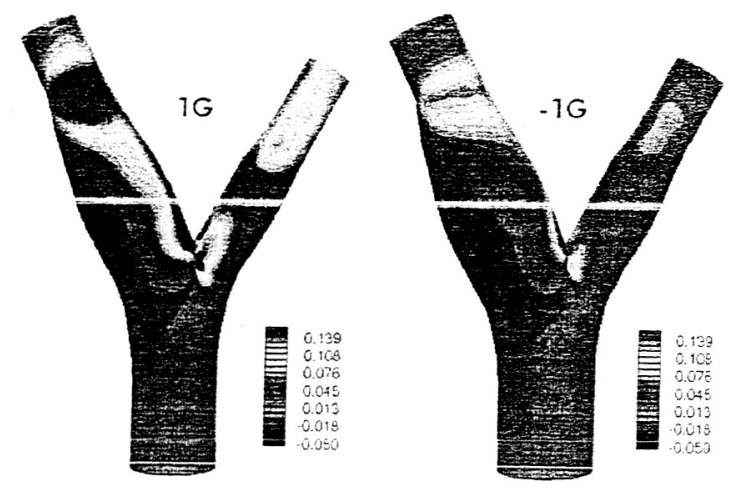

Fig. 13 Gravitational effect on wall shear stress distribution during the systolic deceleration phase.

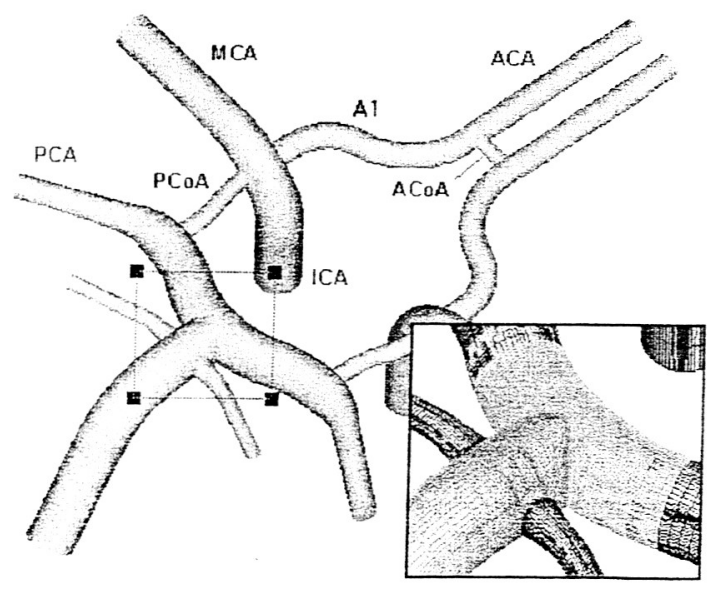

ACA: Anterior Cerebral Artery, ACoA: Anterior Communicating Artery, ICA: Internal Carotid Artery, MCA: Middle Cerebral Artery,

PCA: Posterior Cerebral Artery, PCoA: Posterior Communicating Artery.

Fig. 14 Chimera overset grid with ten domains for an idealized Circle of Willis configuration.

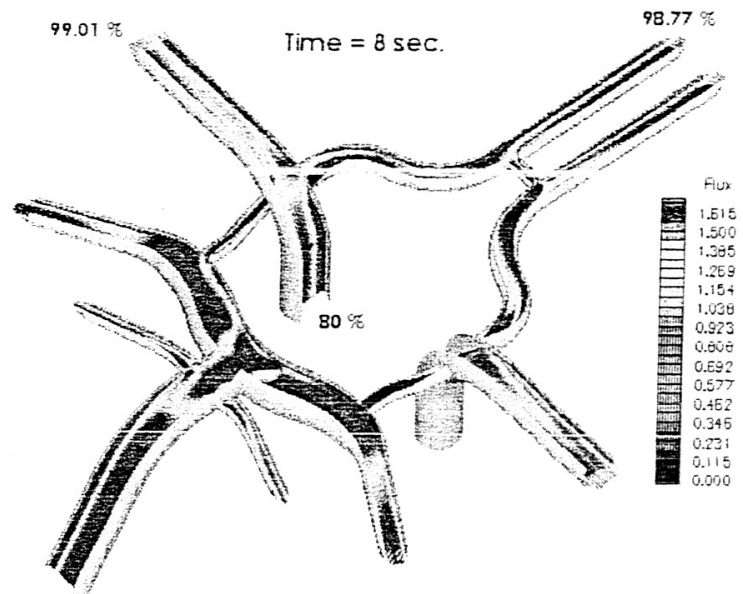

Fig. 15 Collateral circulation with the left internal carotid artery $20 \%$ stenosed under auto-regulation.

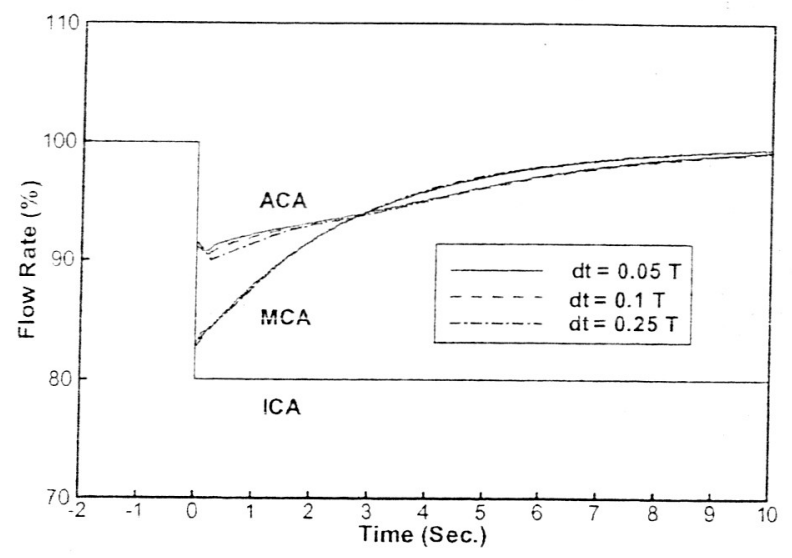

Fig. 16 Percent changes of flow rate in left middle and anterior cerebral arteries under auto-regulation. 


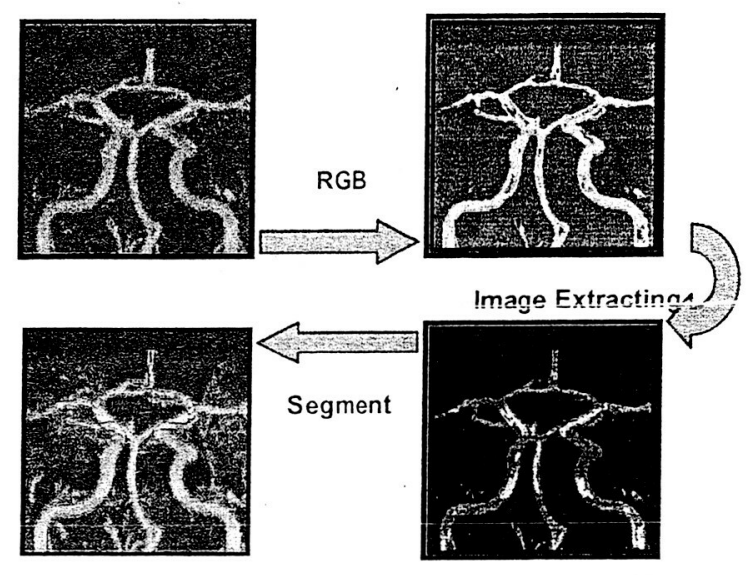

Fig. 17 Image segmentation from a magnetic resonance image for a human-specific Circle of Willis. (MRA provided by $T$. David)

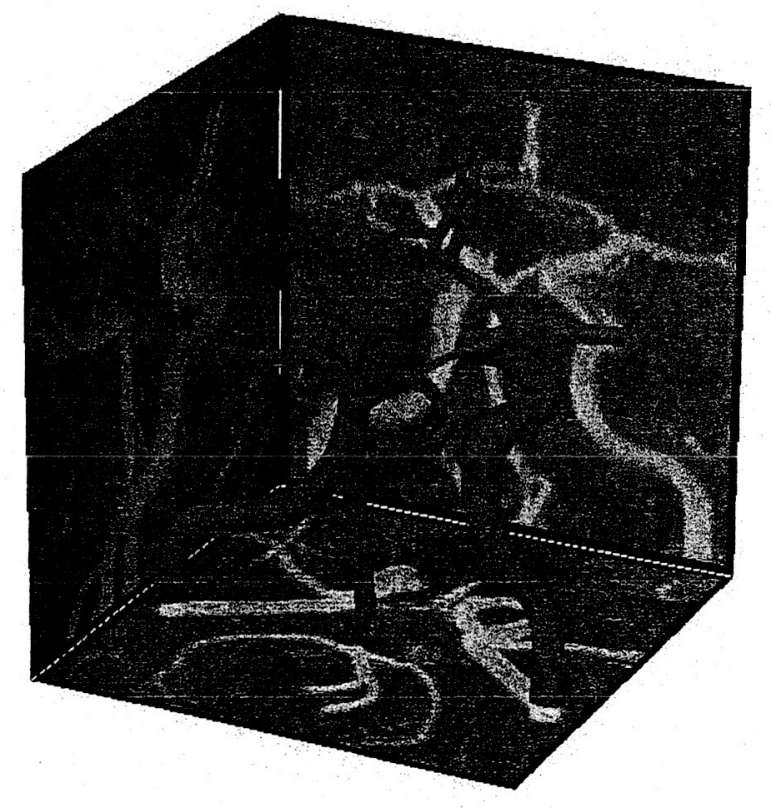

Fig. 18 Three-dimensional reconstruction of an anatomical Circle of Willis configuration.

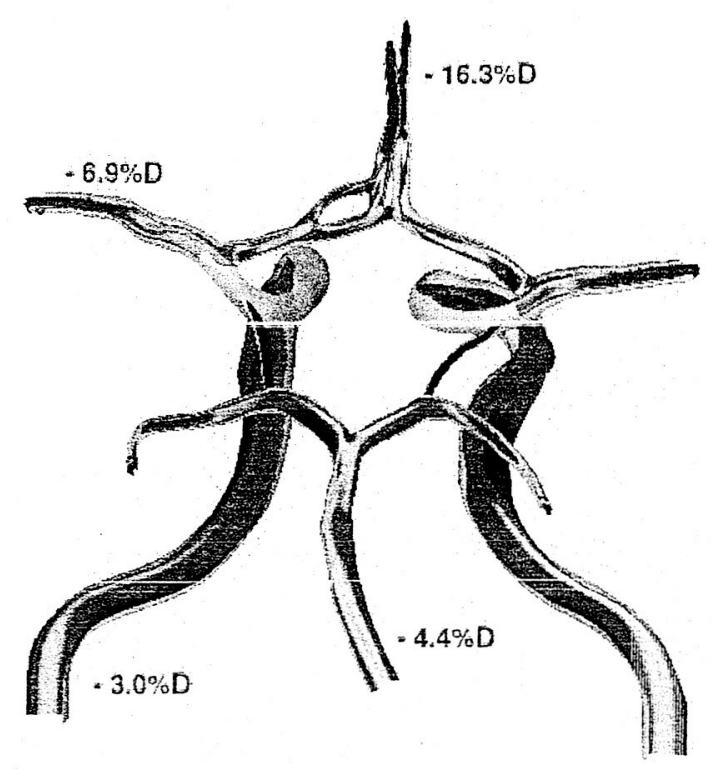

Fig. 19 Time-averaged blood flow within compliant walls in standing posture (1G).

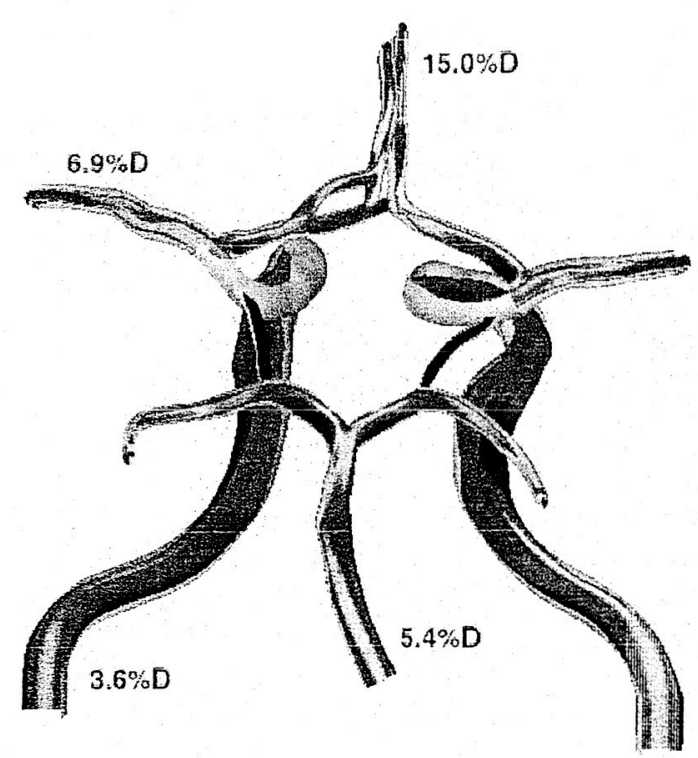

Fig. 20 Time-averaged blood flow within compliant walls in hand-standing posture $(-1 G)$. 